\title{
White blood cell and platelet indices as prognostic markers in patients with invasive ductal breast carcinoma
}

\author{
DIMITRIOS MANTAS, IOANNIS D. KOSTAKIS, NIKOLAOS MACHAIRAS and CHRISTOS MARKOPOULOS
}

Breast Unit, Second Department of Propaedeutic Surgery, Athens Medical School, University of Athens, 11527 Athens, Greece

Received April 1, 2016; Accepted June 8, 2016

DOI: $10.3892 / \mathrm{ol} .2016 .4760$

\begin{abstract}
A growing body of evidence suggests that oncogenesis is associated with systemic inflammation. The present study investigated white blood cell and platelet indices, whose values change during the inflammatory response, in women with invasive ductal breast carcinoma. Preoperatively obtained white blood cell and platelet counts from 53 patients with early breast cancer, who developed systemic metastases over a mean follow-up period of 65 months, were analyzed and compared with those of a matching control group formed of 37 patients with the same characteristics, who remained recurrence-free during the same time period. Patients who developed distant metastasis had a significantly higher mean platelet volume and lower neutrophil count than patients who did not present with distant metastasis. Furthermore, time to distant metastasis development was longer in patients with a lower mean platelet volume, whilst patients with a lower neutrophil count had a shorter systemic disease-free time interval. However, receiver operating characteristic curve analysis demonstrated that these parameters provided moderate accuracy in predicting which patients may develop distant metastasis. No differences were detected between patient groups regarding additional parameters. Patients who developed systemic disease during a mean follow-up period of 65 months were observed to have an increased mean platelet volume and decreased neutrophil count preoperatively. These results indicate that such parameters may be of prognostic value in patients with breast cancer. Studies with a larger number of patients are required to further investigate this hypothesis.
\end{abstract}

\section{Introduction}

Breast cancer represents a major health problem and is the most common malignancy among women, with 400,000 new cases being diagnosed each year worldwide (1). A total of

Correspondence to: Mr. Dimitrios Mantas, Breast Unit, Second Department of Propaedeutic Surgery, Athens Medical School, University of Athens, 17 Agiou Thoma Street, 11527 Athens, Greece E-mail:dvmantas@med.uoa.gr

Key words: breast cancer, metastasis, platelets, white blood cells
232,000 women were diagnosed with breast cancer in 2014 in the United States, corresponding to $28.6 \%$ of all cancer incidents among women (1). Breast cancer is responsible for 40,000 mortalities annually and the lifetime risk of succumbing to the disease is $\sim 3.4 \%$ (1). Despite novel approaches in the therapeutic management of breast cancer, including surgical treatment, and additional chemotherapy and radiation therapy, metastatic disease remains a great clinical challenge, and the therapeutic arsenal remains inadequate in impeding relapse and metastasis. Therefore, novel predictive markers are required to identify high-risk patients who may develop metastases during postoperative surveillance, which may permit oncologists to utilize more efficient patient-tailored treatment strategies.

Systemic inflammatory response has been identified to affect survival in a number of malignancies $(2,3)$. White blood cells are key mediators in this response (2), in addition to platelets, which are involved in mechanisms promoting tumor growth and metastasis (4-6). Various white blood cell and platelet indices, including neutrophil-to-lymphocyte ratio, platelet count, mean platelet volume and platelet-to-lymphocyte ratio, have been reported as potential markers for predicting the progression or recurrence of disease and/or overall survival in several forms of cancer (7-10). The present study aimed to investigate whether changes in white blood cell and platelet indices possess prognostic value regarding the development of distant metastases in patients with newly diagnosed breast cancer.

\section{Patients and methods}

Patients. A total of 90 female patients (mean age, 58.6 years; range, 28-86 years) with newly diagnosed, non-metastatic invasive ductal breast carcinoma, who were treated in the Breast Unit, Second Department of Propaedeutic Surgery, Athens Medical School, University of Athens (Athens, Greece) between February 2005 and December 2014, were included in the current study. Of these patients, 53 (mean age, 55.9 years; range, 28-84 years) developed metastasis at a distant site during the surveillance period and were categorized as the metastasis group, while 37 patients (mean age, 62.5 years; range, 31-86 years) did not develop distant metastasis during surveillance and were used as a control group. The two groups were matched for age, disease stage and duration of surveillance. Data regarding white blood cell count, neutrophil count, neutrophil percentage, lymphocyte count, lymphocyte 
percentage, neutrophil-to-lymphocyte ratio, platelet count, mean platelet volume, platelet distribution width, plateletcrit, platelet-to-neutrophil ratio and platelet-to-lymphocyte ratio was retrospectively collected from complete blood count tests performed upon admission to the Breast Unit. In addition, clinical data concerning age, type of surgery and development of distant metastasis during the follow-up period was obtained, along with pathological data regarding tumor diameter, extent of primary tumor infiltration (T), number of infiltrated lymph nodes, degree of lymph node infiltration $(\mathrm{N})$, tumor-node-metastasis stage (11), grade, presence or absence of lymphovascular invasion, and status of estrogen receptors, progesterone receptors and c-erb-B2 expression, which was collected from the pathology reports of excised specimens. Clinicopathological parameters of the patients are listed in Table I. The present study conforms to the Declaration of Helsinki.

Complete blood count measurement. A total of $3 \mathrm{ml}$ blood was collected in EDTA Vacutainer ${ }^{\circledR}$ Tubes from each patient. All specimens were processed using an XT-4000i Automated Hematology Analyzer (Sysmex Corporation, Kobe, Japan) within 30 min of the blood being drawn.

Statistical analysis. Normality of data distribution was assessed using the Shapiro-Wilk test. Two group comparisons were performed using the $t$-test or the Mann-Whitney $\mathrm{U}$ test when data were normally or not normally distributed, respectively. The optimal cut-off points of parameters that provided significant differences were assessed with the use of receiver operating characteristic (ROC) curves. These cut-off points were taken into account for the calculation of specificity, sensitivity, negative predictive value (NPV), positive predictive value (PPV) and accuracy. For the assessment of time until metastasis development, patients were divided into 4 groups according to the levels of each white blood cell and platelet parameter: Group $1, \leq 25$ th percentile; group $2,>25$ th and $\leq 50$ th percentile; group $3,>50$ th and $\leq 75$ th percentile; group $4,>75$ th percentile. The Kaplan-Meier method was used for the estimation of time to metastasis development and comparisons among groups according to each tested parameter. Multivariate survival analysis was performed with the use of Cox regression with the forward conditional method. All the tests were two-tailed and $\mathrm{P}<0.05$ was considered to indicate a statistically significant difference.

\section{Results}

Complete blood count test results according to the development of secondary distant metastasis. The only parameters that appeared to differentiate between the patients who developed metastasis and those who did not were the mean platelet volume and neutrophil count. Specifically, patients who presented with distant metastasis during the follow-up period had an increased mean platelet volume [mean \pm standard deviation (SD), $10.9 \mathrm{fl} \pm 0.9$ ] in comparison with patients who did not (mean $\pm \mathrm{SD}, 10.1 \mathrm{fl} \pm 1.5)(\mathrm{P}=0.004)$ (Fig. 1). Furthermore, patients who presented with distant metastasis during the surveillance period had decreased neutrophil counts (median, 3,640 U/ $\mu 1$; range, 1,120-8,090 U/ $\mu \mathrm{l}$ ) compared
Table I. Clinicopathological data of patients with invasive ductal breast carcinoma.

\begin{tabular}{lc}
\hline Parameters & $\mathrm{n}, \%$ \\
\hline Tumor stage & \\
T1 & $28(31.1)$ \\
T2 & $45(50.0)$ \\
T3 & $9(10.0)$ \\
T4 & $8(8.9)$
\end{tabular}

Lymph node infiltration

NO

N1

$\mathrm{N} 2$

N3

TNM stage

I

II

$33(36.7)$

III

Tumor grade

Low

$49(54.4)$

High

$41(45.6)$

Estrogen receptor expression

Positive

Negative

Progesterone receptor expression

Positive

$46(51.1)$

Negative

$44(48.9)$

c-erb-B2 expression

Positive

$49(54.4)$

Negative

$41(45.6)$

${ }^{\text {a }}$ th edition of the TNM staging system (11). TNM, tumor-node-metastasis.

with patients who did not present with metastasis (median, 3,950 U/ $\mu \mathrm{l}$; range, 2,100-11,800 U/ $\mu \mathrm{l})(\mathrm{P}=0.0495)$ (Fig. 2). By contrast, no significant differences were observed between the patients with and without distant metastasis with regards to white blood cell count $(\mathrm{P}=0.114)$, neutrophil percentage $(\mathrm{P}=0.071)$, lymphocyte count $(\mathrm{P}=0.571)$, lymphocyte percentage $(\mathrm{P}=0.207)$, neutrophil-to-lymphocyte ratio $(\mathrm{P}=0.191)$, platelet count $(\mathrm{P}=0.116)$, platelet distribution width $(\mathrm{P}=0.187)$, plateletcrit $(\mathrm{P}=0.669)$, platelet-to-neutrophil ratio $(\mathrm{P}=0.211)$ or platelet-to-lymphocyte ratio $(\mathrm{P}=0.664)$.

ROC curve analysis and calculation of sensitivity, specificity, $P P V, N P V$ and accuracy. Regarding mean platelet volume, ROC curve analysis provided an area under the curve (AUC) of 0.674 [95\% confidence interval (CI): 0.553-0.795; standard error (SE): 0.062; $\mathrm{P}=0.006]$ and an optimal cut-off point of $10 \mathrm{fl}$ (>10 fl sensitivity, $80.4 \%$; specificity, 52.8\%; PPV, 70.7\%; NPV, 65.5\%; accuracy, 69\%) (data not shown). Regarding neutrophil count, ROC curve analysis provided an AUC of 0.622 (95\% CI, 0.506-0.738; SE, 0.059; P=0.0495) 


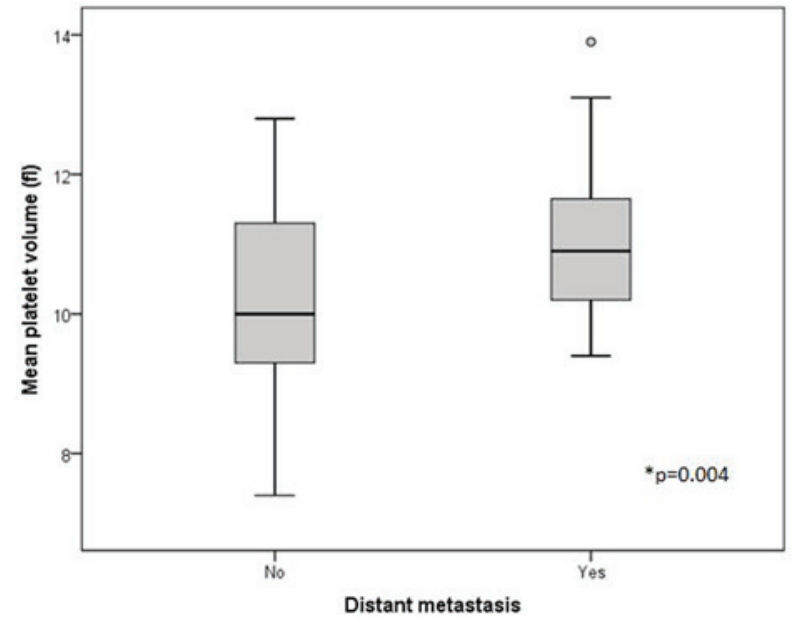

Figure 1. Mean platelet volume in patients who developed distant metastasis and patients who did not. Circles indicate extreme values/outliers.

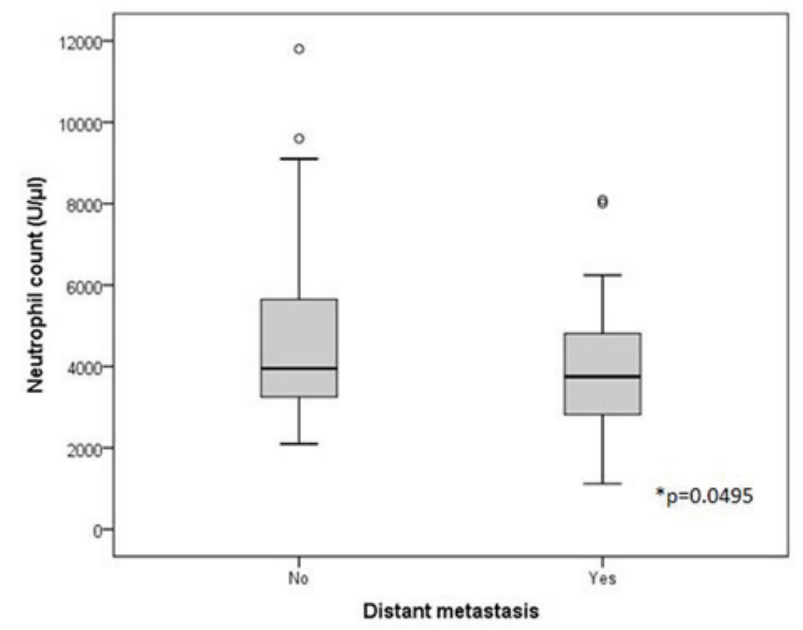

Figure 2. Neutrophil count in patients who developed distant metastasis and patients who did not. Circles indicate extreme values/outliers.

and an optimal cut-off point of 4,000 $\mathrm{U} / \mu 1(<4,000 \mathrm{U} / \mu 1$ sensitivity, 60.4\%; specificity, 51.4\%; PPV, 64\%; NPV, 47.5\%; accuracy, 56.7\%) (data not shown).

Survival analysis. The mean and the median period of surveillance was 65 months (range, 3-121 months). When group 1 ( $\leq 25$ th percentile) was compared with groups 2 , 3 and 4 ( $>25$ th percentile) for each parameter, significant differences were observed in mean platelet volume and neutrophil count. In particular, patients with a lower mean platelet volume (group 1) experienced a longer period of time to distant metastasis development than patients with a higher mean platelet volume (groups 2, 3 and 4) $[\mathrm{P}=0.001$; group 1: Mean time $\pm \mathrm{SE}, 65 \pm 5$ months (95\% CI, 55.2-74.9); groups 2, 3 and 4: Mean time \pm SE, 41.6 \pm 3.1 months $(95 \%$ CI, 35.4-47.7)] (Fig. 3). In addition, patients with a lower neutrophil count (group 1) experienced a shorter time to distant metastasis development than patients with a higher neutrophil count (groups 2, 3 and 4) [P=0.001; group 1: Mean time \pm SE, 33.6 \pm 4.7 months (95\% CI, 24.3-42.9); groups 2, 3 and 4: Mean time \pm SE, 52.5 \pm 3.4 months (95\% CI, 45.9-59.1)]

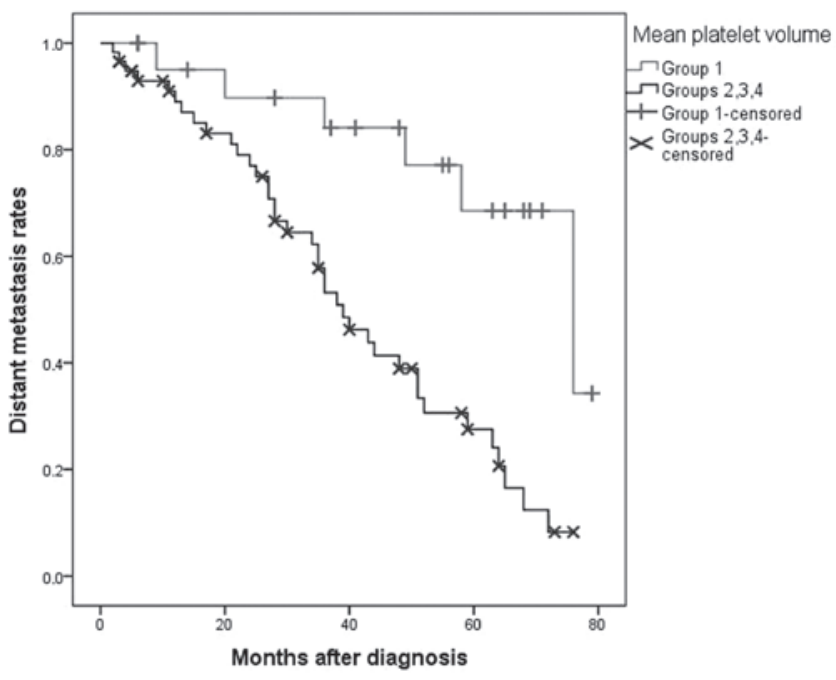

Figure 3. Distant metastasis rates in patients with lower (group 1) and higher (groups 2,3 and 4) mean platelet volume.

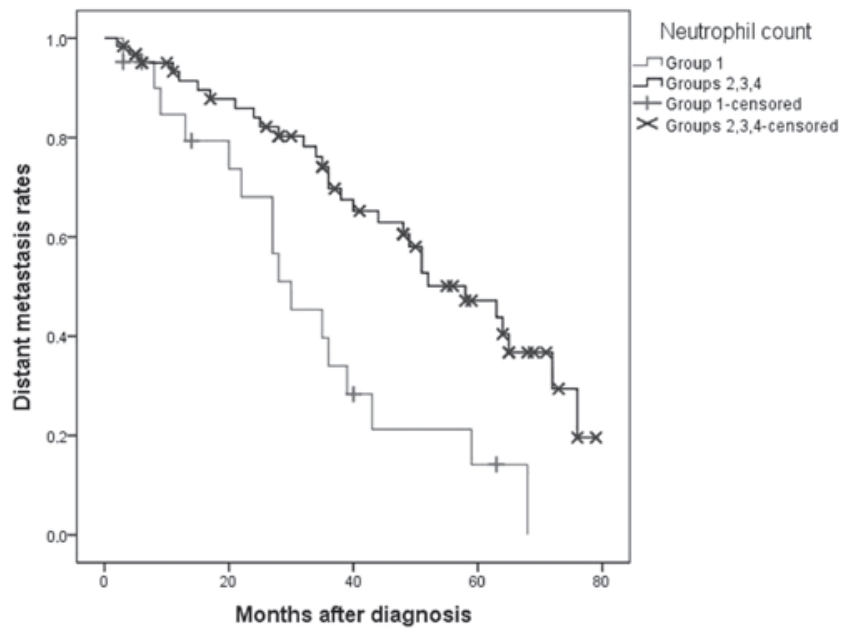

Figure 4. Distant metastasis rates in patients with lower (group 1) and higher (groups 2,3 and 4) neutrophil counts.

(Fig. 4). By contrast, no significant differences were detected between the patients with and without distant metastasis regarding white blood cell count $(\mathrm{P}=0.105)$, neutrophil percentage $(\mathrm{P}=0.17)$, lymphocyte count $(\mathrm{P}=0.587)$, lymphocyte percentage $(\mathrm{P}=0.108)$, neutrophil-to-lymphocyte ratio $(\mathrm{P}=0.34)$, platelet count $(\mathrm{P}=0.17)$, platelet distribution width $(\mathrm{P}=0.104)$, plateletcrit $(\mathrm{P}=0.683)$, platelet-to-neutrophil ratio $(\mathrm{P}=0.647)$ or platelet-to-lymphocyte ratio $(\mathrm{P}=0.433)$. However, the multivariate survival analysis provided high $\mathrm{T}(\mathrm{P}=0.0002)$ and high grade $(\mathrm{P}=0.001)$ as independent risk factors for future development of distant metastasis, but not mean platelet volume $(\mathrm{P}=0.096)$ or neutrophil count $(\mathrm{P}=0.993)$.

\section{Discussion}

There is a growing body of evidence to suggest that immune cells and platelets are implicated in complex crosstalk with malignant cells and their microenvironment in various malignancies $(2,4-6)$. Regarding white blood cells, increased tumor 
infiltration by inflammatory cells has been associated with favorable histopathological features and prognosis in numerous types of cancer (2). Furthermore, it has been observed that platelets interact with malignant cells and suppress the inflammatory response against them. In addition, platelets secrete various growth factors and cytokines that promote angiogenesis, tumor growth, invasion and metastasis either directly or indirectly (4-6).

Several clinical studies (7-10) have aimed to evaluate whether these interactions between white blood cells and platelets on one side and tumor cells on the other side may cause measurable alterations in blood parameters, as they are depicted in the complete blood count test. Furthermore, studies have attempted to detect any potential diagnostic and/or prognostic values of blood test parameters (7-10). Parameters that have been examined regarding breast cancer include mean platelet volume, platelet-to-lymphocyte ratio and neutrophil-to-lymphocyte ratio.

Neutrophil-to-lymphocyte ratio is the most studied parameter in breast cancer. In particular, Ozyalvacli et al (12) observed that neutrophil-to-lymphocyte ratio was increased in patients with breast cancer compared with patients with benign proliferative breast disease. In addition, Dirican et al (13) and Jia et al (14) reported that patients with breast cancer with a low neutrophil-to-lymphocyte ratio present longer disease-free and overall survival times than those who have a high neutrophil-to-lymphocyte ratio. Similar results have been yielded by several studies (15-19), which concluded that patients with a high pretreatment neutrophil-to-lymphocyte ratio have higher 5-year mortality rates than patients with a low pretreatment neutrophil-tolymphocyte ratio. By contrast, Ulas et al (20) did not detect any significant associations between clinicopathological parameters or survival rate and neutrophil-to-lymphocyte ratio in patients with breast cancer.

Regarding platelet-to-lymphocyte ratio in patients with breast cancer, several studies have also attempted to identify any potential prognostic value. Patients with a higher platelet-to-lymphocyte ratio experienced poorer survival times $(15,16,21)$ and a larger number of infiltrated lymph nodes (22) compared with patients with a lower platelet-to-lymphocyte ratio. By contrast, Ulas et al (20) and Yao et al (18) reported that platelet-to-lymphocyte ratio does not significantly affect survival times in patients with breast cancer.

Gu et al (23) reported that mean platelet volume is higher in patients with breast cancer than in patients with benign breast tumors. Additionally, it was observed that increased mean platelet volume was associated with larger tumors, higher stage, distant metastases and a poorer prognosis in patients with breast cancer (23). By contrast, Yao et al (18) reported that there was no significant difference in survival between higher and lower mean platelet volumes.

The present study aimed to evaluate the potential prognostic role of white blood cell and platelet indices in determining which high-risk patients with newly diagnosed invasive ductal breast carcinoma are likely develop distant metastasis in the future. According to the results, patients who are predicted to develop distant metastasis have a higher mean platelet volume and lower neutrophil count in comparison with those who are unlikely to develop metastasis. This is also confirmed by survival analysis, which demonstrated that patients with a lower mean platelet volume had a longer time to metastasis development, whereas patients with a lower neutrophil count had a shorter time to metastasis development. However, these two parameters yielded only moderate sensitivity and specificity in identifying the patients with breast cancer who will develop distant metastasis.

In conclusion, mean platelet volume and neutrophil count are two blood test parameters that may be useful in identifying patients with newly diagnosed invasive ductal breast carcinoma that are likely to present with distant metastasis in the future. Nevertheless, further studies are required to confirm the findings of the current study, which may allow the construction of a predictive model for patients with breast cancer, including mean platelet volume and neutrophil count, amongst other blood test parameters.

\section{References}

1. Siegel R, Ma J, Zou Z and Jemal A: Cancer statistics, 2014. CA Cancer Clin 64: 9-29, 2014.

2. Giraldo NA, BechtE, Vano Y, Sautès-Fridman C and Fridman WH: The immune response in cancer: From immunology to pathology to immunotherapy. Virchows Arch 467: 127-135, 2015.

3. Wu Y, Fu X, Zhu X, He X, Zou C, Han Y, Xu M, Huang C, Lu X and Zhao Y: Prognostic role of systemic inflammatory response in renal cell carcinoma: A systematic review and meta-analysis. J Cancer Res Clin Oncol 137: 887-896, 2011.

4. Menter DG, Tucker SC, Kopetz S, Sood AK, Crissman JD and Honn KV: Platelets and cancer: A casual or causal relationship: Revisited. Cancer Metastasis Rev 33: 231-269, 2014.

5. Yan M and Jurasz P: The role of platelets in the tumor microenvironment: From solid tumors to leukemia. Biochim Biophys Acta 1863: 392-400, 2016.

6. Franco AT, Corken A and Ware J: Platelets at the interface of thrombosis, inflammation, and cancer. Blood 126: 582-588, 2015.

7. Templeton AJ, McNamara MG, Šeruga B, Vera-Badillo FE, Aneja P, Ocaña A, Leibowitz-Amit R, Sonpavde G, Knox JJ, Tran B, et al: Prognostic role of neutrophil-to-lymphocyte ratio in solid tumors: A systematic review and meta-analysis. J Natl Cancer Inst 106: dju124, 2014.

8. Paramanathan A, Saxena A and Morris DL: A systematic review and meta-analysis on the impact of pre-operative neutrophil lymphocyte ratio on long term outcomes after curative intent resection of solid tumours. Surg Oncol 23: 31-39, 2014.

9. Xin-Ji Z, Yong-Gang L, Xiao-Jun S, Xiao-Wu C, Dong Z and Da-Jian Z: The prognostic role of neutrophils to lymphocytes ratio and platelet count in gastric cancer: A meta-analysis. Int J Surg 21: 84-91, 2015.

10. Templeton AJ, Ace O, McNamara MG, Al-Mubarak M, Vera-Badillo FE, Hermanns T, Seruga B, Ocaña A, Tannock IF and Amir E: Prognostic role of platelet to lymphocyte ratio in solid tumors: A systematic review and meta-analysis. Cancer Epidemiol Biomarkers Prev 23: 1204-1212, 2014.

11. Sobin LH, Gospodarowicz MK and Wittekind CH (ed): Breast tumours. In: TNM Classification of Malignant Tumours. 7th edition. Wiley-Blackwell, Chichester, West Sussex, UK, pp181-193, 2010.

12. Ozyalvacli G, Yesil C, Kargi E, Kizildag B, Kilitci A and Yilmaz F: Diagnostic and prognostic importance of the neutrophil lymphocyte ratio in breast cancer. Asian Pac J Cancer Prev 15: 10363-10366, 2014.

13. Dirican A, Kucukzeybek BB, Alacacioglu A, Kucukzeybek Y, Erten C, Varol U, Somali I, Demir L, Bayoglu IV, Yildiz Y, et al: Do the derived neutrophil to lymphocyte ratio and the neutrophil to lymphocyte ratio predict prognosis in breast cancer? Int J Clin Oncol 20: 70-81, 2015.

14. Jia W, Wu J, Jia H, Yang Y, Zhang X, Chen K and Su F: The peripheral blood neutrophil-to-lymphocyte ratio is superior to the lymphocyte-to-monocyte ratio for predicting the long-term survival of triple-negative breast cancer patients. PLoS One 10: e0143061, 2015. 
15. Azab B, Shah N, Radbel J, Tan P, Bhatt V, Vonfrolio S, Habeshy A, Picon A and Bloom S: Pretreatment neutrophil/lymphocyte ratio is superior to platelet/lymphocyte ratio as a predictor of long-term mortality in breast cancer patients. Med Oncol 30: 432, 2013.

16. Koh CH,Bhoo-Pathy N,Ng KL,JabirRS, Tan GH,See MH,Jamaris S and Taib NA: Utility of pre-treatment neutrophil-lymphocyte ratio and platelet-lymphocyte ratio as prognostic factors in breast cancer. Br J Cancer 113: 150-158, 2015.

17. Noh H, Eomm M and Han A: Usefulness of pretreatment neutrophil to lymphocyte ratio in predicting disease-specific survival in breast cancer patients. J Breast Cancer 16: 55-59, 2013.

18. Yao M, Liu Y, Jin H, Liu X, Lv K, Wei H, Du C, Wang S, Wei B and Fu P: Prognostic value of preoperative inflammatory markers in Chinese patients with breast cancer. Onco Targets Ther 7: 1743-1752, 2014.

19. Koh YW, Lee HJ, Ahn JH, Lee JW and Gong G: Prognostic significance of the ratio of absolute neutrophil to lymphocyte counts for breast cancer patients with ER/PR-positivity and HER2-negativity in neoadjuvant setting. Tumour Biol 35: 9823-9830, 2014.
20. Ulas A, Avci N, Kos T, Cubukcu E, Olmez OF, Bulut N and Degirmenci $\mathrm{M}$ : Are neutrophil/lymphocyte ratio and platelet/lymphocyte ratio associated with prognosis in patients with HER2-positive early breast cancer receiving adjuvant trastuzumab? J BUON 20: 714-722, 2015.

21. Krenn-Pilko S, Langsenlehner U, Thurner EM, Stojakovic T, Pichler M, Gerger A, Kapp KS and Langsenlehner T: The elevated preoperative platelet-to-lymphocyte ratio predicts poor prognosis in breast cancer patients. Br J Cancer 110: 2524-2530, 2014.

22. Seretis C, Seretis F, Lagoudianakis E, Politou M, Gemenetzis G and Salemis NS: Enhancing the accuracy of platelet to lymphocyte ratio after adjustment for large platelet count: A pilot study in breast cancer patients. Int J Surg Oncol 2012: 653608, 2012.

23. Gu M, Zhai Z, Huang L, Zheng W, Zhou Y, Zhu R, Shen F and Yuan C: Pre-treatment mean platelet volume associates with worse clinicopathologic features and prognosis of patients with invasive breast cancer. Breast Cancer: Aug 26, 2015 (Epub ahead of print). 